\title{
Soil Water Sensor Performance and Corrections with Multiple Installation Orientations and Depths under Three Agricultural Irrigation Treatments
}

\author{
Yong Chen ${ }^{1, *}$, Gary W. Marek ${ }^{2}$, Thomas H. Marek ${ }^{3}$, Kevin R. Heflin ${ }^{3}$, Dana O. Porter ${ }^{4}$, \\ Jerry E. Moorhead ${ }^{2}$ and David K. Brauer ${ }^{2}$ \\ 1 Department of Ecosystem Science and Management, Texas A\&M University, College Station, TX 77843, USA \\ 2 USDA-ARS Conservation and Production Research Laboratory, 300 Simmons Rd., Unit 10, Bushland, TX \\ 79012, USA \\ 3 Texas A\&M AgriLife Research and Extension Center at Amarillo, 6500 Amarillo Blvd. W., Amarillo, TX \\ 79106, USA \\ 4 Texas A\&M AgriLife Research and Extension Center at Lubbock, 1102 E FM 1294, Lubbock, TX 79403, USA \\ * Correspondence: yongchen@neo.tamu.edu
}

Received: 9 May 2019; Accepted: 24 June 2019; Published: 28 June 2019

\begin{abstract}
Performance evaluations and corrections of soil water sensors have not been studied using different installation orientations under various irrigation treatments in the Texas High Plains. This study evaluated the performance of four sensors using factory calibration and derived field corrections as compared to calibrated neutron moisture meters (NMMs). Sensor performance was assessed using horizontal insertion, laid horizontal placement, and vertical insertion at 15.2, 45.7, and $76.2 \mathrm{~cm}$ depths in a clay loam soil with three irrigation treatments. Results indicated the factory-calibrated Acclima $315 \mathrm{~L}$ performed satisfactorily using horizontal insertion as compared to NMM measurements at 45.7 and $76.2 \mathrm{~cm}$ depths with a $\pm 2 \%$ mean difference (MD) and $<3.5 \%$ root mean square error (RMSE). The factory-calibrated Acclima $315 \mathrm{~L}$ using horizontal insertion also performed satisfactorily across all irrigation treatments according to soil profile water storage $(\mathrm{MD}=0.36 \%$ and RMSE $=3.25 \%)$. Generally, the factory-calibrated Decagon GS1 and Campbell Scientific 655 using vertical insertion agreed more closely with NMM measurements compared with other installation orientations. There was a significant underestimation of water storage $(>60 \mathrm{~mm})$ in the $0.9 \mathrm{~m}$ soil profile using the Watermark 200SS. In summary, field corrections are required for Decagon GS1, Campbell Scientific 655, and Watermark 200SS sensors.
\end{abstract}

Keywords: soil water sensor; soil water measurement; soil water content; neutron moisture meter; corn; semi-arid region; factory calibration; field correction

\section{Introduction}

The semi-arid Southern Great Plains is one of the most productive irrigated agricultural regions in the United States (U.S.). The underlying Ogallala Aquifer serves as a crucial groundwater source for irrigated agricultural crop production. However, intensive irrigation pumping with very little recharge in this region has resulted in a considerable decline in groundwater storage of the Ogallala Aquifer in recent years [1,2]. In addition, competition for water from urban, industrial, and agricultural sectors along with a changing climate has resulted in notable droughts and water shortages, placing agricultural production and accompanying economic prosperity at risk [3]. Therefore, there is an urgent need for research regarding improved agricultural irrigation management practices for the U.S. Southern Great Plains. 
Monitoring of soil water content (SWC) throughout the growing season can facilitate irrigation scheduling [4]. The use of soil water sensors for SWC estimations is gaining widespread state and federal support with cost sharing in the U.S. For instance, the U.S. Department of Agriculture (USDA) awarded the White River Irrigation District in Arkansas \$4.45 million in 2009 to install soil water measurement and monitoring technologies, which included investments in soil water sensors [5]. In addition, several programs and networks were established in the U.S. for soil and climatic observations using different in situ sensors with differing accuracy and precision. For example, the Texas Soil Observation Network (TxSON), West Texas Mesonet, Oklahoma Mesonet, U.S. Climate Reference Network (USCRN), and Soil Climate Analysis Network (SCAN) are collecting SWC data using soil water sensors $[6,7]$. However, several studies have alluded that soil water sensors using factory calibrations report readings that do not adequately represent actual SWC in field conditions [8-13]. In most cases, a field or site-specific correction is required to improve the accuracy of sensor readings for agricultural and research applications [6]. Additionally, due to the vertical variations (stratification) of soil properties and water regimes, it is possible that the soil water sensors installed using different installation orientations may result in different SWC readings. For instance, Plauborg et al. [14] studied the effects of installation orientations of horizontal and vertical insertions on the performance of Campbell Scientific 616 (CS-616) and found that vertically installed CS-616 sensors responded well to changes in SWC when using the factory calibration. However, horizontally installed CS-616 sensors required a site-specific soil correction to obtain a reasonable measurement of SWC. Consequently, a soil-specific sensor correction may be necessary, depending on site-specific conditions and sensor application. However, a lack of such studies was found in the literature [15].

Corn (Zea mays L.) is one of the dominant crops in the semi-arid U.S. Southern Great Plains, which demands a large amount of irrigation water to meet its full crop evapotranspiration (ETc) requirement. Due to the declining groundwater resources and resultant reduced well capacities, deficit irrigation for corn production is increasingly being practiced [16-20], and accurate measurements of SWC are crucial for profitable corn production under limited irrigation strategies. As far as we know, concomitant comparisons of soil water sensor performance under different irrigation level treatments has rarely been studied [21]. In this study, the performance of a soil water potential sensor and three soil water content sensors installed at three depths and using three installation orientations under three irrigation treatments were evaluated against collocated measurements from a calibrated neutron moisture meter (NMM). The primary goal was to assess the accuracy of the soil water sensors using factory calibrations and determine field-based corrections, if needed. Specific objectives of the study were (1) to evaluate the accuracy of each of the four sensor types, installed using three different orientations at three depths, under three irrigation treatment levels, compared against SWC measurements from calibrated NMMs; (2) to compare the water storage in the soil profile derived from integrating the soil water sensor measurements against those integrated from NMM readings; and (3) to explore the field corrections for the four sensors and develop the soil-specific correction curves for use with regional agricultural production applications.

\section{Materials and Methods}

\subsection{Study Area Description}

The study site is located at the Texas A\&M AgriLife Research Emeny Center Pivot Field, Bushland, Texas $\left(35.2^{\circ} \mathrm{N}, 102.1^{\circ} \mathrm{W}\right)$. The elevation of the study site is $\sim 1170 \mathrm{~m}$ above mean sea level, and the slope is $<1 \%$. The regional semi-arid climate has a mean annual precipitation and air temperature of $488 \mathrm{~mm}$ and $14.1^{\circ} \mathrm{C}$. The circular field is irrigated with a center pivot system equipped with low elevation spray application (LESA) nozzles positioned on a $1.5 \mathrm{~m}$ spacing and at a height of $0.5 \mathrm{~m}$ above the ground surface. Three targeted irrigation treatment levels of $100 \%, 75 \%$, and $50 \%$ ETc requirements were used in this study (Figure 1). The soil is a Pullman clay loam soil (fine, mixed, superactive, thermic Torrertic Paleustoll) [22]. The permanent wilting point (PWP) and field capacity (FC) are approximately 0.18 
and $0.33 \mathrm{~m}^{3} \mathrm{~m}^{-3}$, respectively. Measured site-specific soil information by layer is provided in Table S1 in the Supplementary Materials [23,24]. An adjacent research-grade weather station maintained in accordance with the American Society of Civil Engineers-Environmental and Water Resources Institute (ASCE-EWRI) specifications [25] was used to measure meteorological data for this study.

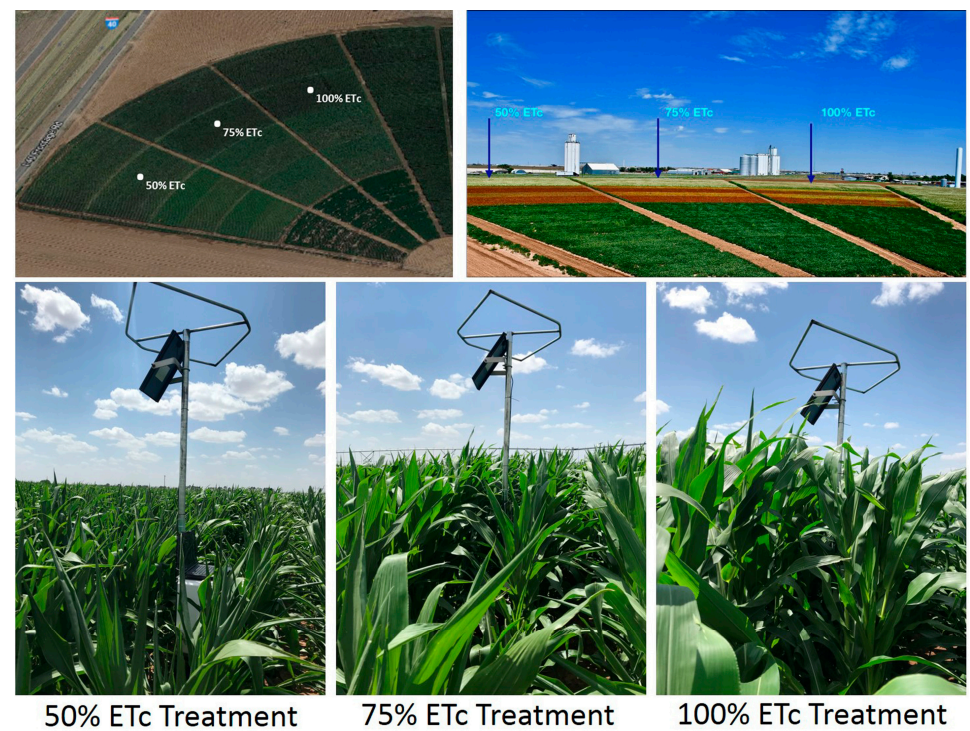

Figure 1. The Texas A\&M AgriLife Research Emeny Center Pivot Field with corn under the three irrigation treatments of $100 \%, 75 \%$, and $50 \%$ crop evapotranspiration (ETc) conditions.

\subsection{Agronomic Management}

Conventional tillage was performed prior to planting using a tandem disk. Fertilizer was applied according to recommendations using soil test data from a commercial soil testing company for a targeted corn grain yield of $\sim 18.83 \mathrm{Mg} \mathrm{ha}^{-1}\left(300 \mathrm{bu} \mathrm{ac}^{-1}\right)$. The field was bedded to a row spacing of 0.76 m (30 in.). A full-season corn hybrid, DuPont Pioneer 1366 AM (113 days-comparative relative maturity), was planted on 18 May 2018 at a rate of 83,275 seeds ha ${ }^{-1}$ (33,700 seeds ac $\left.{ }^{-1}\right)$ using a six-row John Deere computer controlled Max-Emerge planter (Deere \& Co., Moline, IL, USA). Irrigations of $31.75 \mathrm{~mm}$ (1.25 in.) to $38.1 \mathrm{~mm}$ (1.5 in.) for each irrigation event were scheduled to target $100 \%$ of corn ET requirement using a growth-staged crop coefficient and daily reference ET method calculated from the meteorological station data. Deficit irrigation treatments of $75 \%$ and 50\% ETc requirements occurred on the same dates but received $75 \%$ and $50 \%$ of the full irrigation amounts, achieved by increased system speed control (Figure 2). However, full irrigation of all three irrigation treatments was maintained during the early crop development period (30 days after planting) to ensure crop establishment and to satisfy water requirements of early vegetative growth stages. The corn was harvested on 15 November 2018.

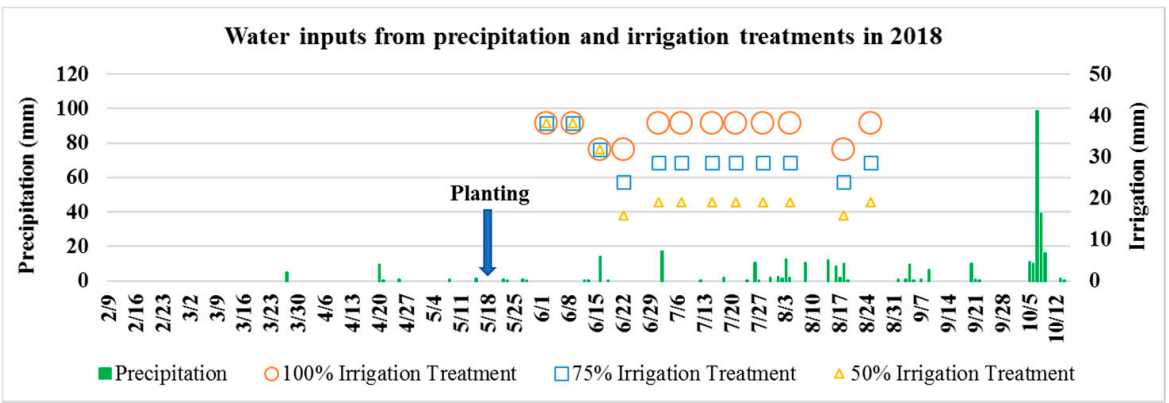

Figure 2. Precipitation and three irrigation treatments of $100 \%, 75 \%$, and $50 \%$ crop evapotranspiration requirements in 2018. 


\subsection{Soil Water Sensor Installation and Layout}

Three trenches measuring $0.5 \mathrm{~m}$ (20 in.) wide by $5.5 \mathrm{~m}$ (18 ft.) long by $0.76 \mathrm{~m}$ (30 in.) deep were excavated in each of the irrigation treatment fields in January 2018. Each trench was centered between two crop beds to allow for installation of the soil sensors on each side of the trench. Sensors were installed at depths of $15.2 \mathrm{~cm}$ (6 in.), $45.7 \mathrm{~cm}$ (18 in.), and $76.2 \mathrm{~cm}$ (30 in.), the midpoints of soil profile depth zones of $0-0.30 \mathrm{~m}(0-1 \mathrm{ft}),. 0.30-0.61 \mathrm{~m}(1-2 \mathrm{ft}$.$) , and 0.61-0.91 \mathrm{~m}(2-3 \mathrm{ft}$.$) . Subsequent surface$ tillage and bedding were conducted by hand to avoid damage to the $15.2 \mathrm{~cm}$ sensors and wiring. Care was taken so that manual tillage and shaping operations would match those of the surrounding field. At planting, corn seed was manually planted at double the field seeding rate in the sensor array area to ensure adequate germination. Following germination, plants in the sensor array area were thinned manually to match the populations of the surrounding fields so that water extraction would be similar. Four commercially available sensors were chosen for evaluation. A soil water potential sensor (Watermark 200SS, Irrometer, Inc., Riverside, CA, USA) and three soil water content sensors, Acclima 315L (Acclima, Inc., Meridian, ID, USA), Decagon GS1 (METER Group, Inc., Pullman, WA, USA), and Campbell Scientific 655 (Campbell Scientific, Inc., Logan, UT, USA), denoted as WM-200SS, ACC-315L, DEC-GS1, and CS-655, respectively, hereafter, were installed and became operational on 8 February 2018.

Sensor installation orientations consisted of vertical insertion into undisturbed soil, laid horizontal placement, and horizontal insertion into undisturbed soil (Figure S1 in the Supplementary Materials). The laid horizontal placement was performed by placing the sensor at the prescribed depths over undisturbed soil, either at the bottom of the trench $(76.2 \mathrm{~cm})$ or over shelves cut into the trench sidewalls (15.2 and $45.7 \mathrm{~cm}$ ), followed by manually backfilling soil over the sensor. The horizontal insertion orientation for sensors with rod electrodes was achieved by inserting the sensor into the undisturbed soil sidewall at each of the three depths. Vertical insertion was achieved by inserting sensors into undisturbed soil into shelves cut into the trench sidewalls. Vertical and horizontal insertion of the WM-200SS sensors was achieved by boring a slightly oversized hole into undisturbed soil, followed by insertion using a soil slurry. It is acknowledged that the manufacturers of the WM-200SS do not recommend installation orientations other than vertical. However, all installation orientations were performed for all sensor types to address questions from area producers and crop consultants regarding sensor installation procedures and orientations and potential effects on output values. Duplicate sensors for each sensor type, orientation, and depth were installed in each trench at each location. The average of the two sensor values from each replication was used to conduct the analyses in this study. In the case of erroneous or missing data, a singular value from one sensor was used for analysis. Seventy-two sensors in total were installed (18 each of the four study sensors) in each trench, resulting in sensors being positioned approximately every $20 \mathrm{~cm}$ horizontally along the trench sidewalls. The trench was backfilled and water packed using the excavated soil. Crop beds and furrows were then manually constructed within the trench area to align them with those of the circular rows of the field. Sensors were polled every five minutes and data were compiled into 15-min average values. The data collection instrumentation consisted of a Campbell Scientific CR6 datalogger coupled with an AM 16/32 multiplexer for each of the three treatment plots.

Two NMM access tubes were installed adjacent to each end of the sensor array in undisturbed soil for each ETc level. Readings from the NMM were used for comparison with values from the soil water sensors in this study. In general, the NMM readings were taken twice weekly, one or two days prior to an irrigation event, and two days following an irrigation or precipitation. This approach was designed to capture SWC readings from both relatively wet and dry soil conditions.

\subsection{Sensor Performance Comparisons}

Sensor performance was assessed by comparing statistical parameters using two approaches: (1) Comparison between the average SWC of the sensor pairs and the NMM measurements at each installation depth (i.e., point comparisons), and (2) comparison of water storage in the soil 
profile as determined by integration of soil water sensor values and the NMM measurements. Direct comparison between depth-specific sensor measurements and NMM readings is known to be problematic. Bell et al. [26] and Schwartz et al. [27] cautioned against making direct comparisons between depth-specific SWC readings from NMMs and collocated soil water sensor measurements due to the large differences in the sensing volume of the NMM $\left(2.8-3.4 \times 10^{5} \mathrm{~cm}^{3}\right)$ compared to that of most soil water sensors $\left(<500 \mathrm{~cm}^{3}\right)$. This difference in soil sensing volumes can result in significantly different SWC readings as relatively small inconsistencies in soil properties and conditions can be overstated by a small sensing volume, but lessened, or averaged out by a larger sensing volume. Therefore, point measurements from the soil sensor array were integrated to estimate the profile water storage for comparison with the corresponding NMM measurements.

In this study, water storage of the soil profile to a depth of $0.9 \mathrm{~m}$ was determined by integrating both soil water measurements of the respective sensor at the three depths and from NMM readings at depths of $0.1,0.3,0.5$, and $0.7 \mathrm{~m}$ with using a half weighted value for the $0.9 \mathrm{~m}$ depth $[27,28]$. Specifically, the soil profile water storage determined by NMM readings was calculated using 2/9 weighted value at the depths of $0.1,0.3,0.5$, and $0.7 \mathrm{~m}$ and using a $1 / 9$ weighted value of the $0.9 \mathrm{~m}$ depth. A depth of $0.9 \mathrm{~m}$ is generally deemed as the manageable irrigation profile depth during the growing season for the clay loam soil prevalent within the region. Statistical analysis included descriptive statistics and analyses of variance $(p<0.05)$ conducted using SPSS 22.0 software (IBM SPSS Statistics, IBM Corporation, Armonk, NY, USA) to detect differences between sensor and NMM measurements. Three statistical parameters were used to evaluate the performance of the soil water sensors as compared to the NMM measurements.

The mean difference (MD) is the average difference between sensor and NMM measurements [29], calculated as:

$$
\mathrm{MD}=\frac{\sum_{\mathrm{i}=1}^{\mathrm{n}}(\mathrm{Msi}-\mathrm{Mni})}{\mathrm{n}}
$$

where $M_{s i}$ is the ith measurement obtained through a sensor; $M_{n i}$ is the ith measurement acquired by a $\mathrm{NMM}$; and $\mathrm{n}$ is the sample size.

The root mean square error (RMSE) is the total difference between a sensor and the corresponding NMM measurements of the soil water content [30], calculated as:

$$
\mathrm{RMSE}=\left[\frac{1}{\mathrm{n}} \sum_{\mathrm{i}=1}^{\mathrm{n}}(\mathrm{Msi}-\mathrm{Mni})^{2}\right]^{0.5}
$$

The standard deviation (SD) is a measure that is used to quantify the amount of variation or dispersion of a set of data values [31], calculated as:

$$
\mathrm{SD}=\sqrt[2]{\mathrm{RMSE}^{2}-\mathrm{MD}^{2}}
$$

The coefficient of determination $\left(R^{2}\right)$ was used to demonstrate the degree of association between sensor measurements and NMM readings. Linear regression was performed to compute the slope and intercept values [32,33]. Linear, exponential, logarithmic, and quadratic correction equations were used for the sensor field corrections. Values of MD, RMSE, and SD approaching zero and $R^{2}$ approaching a value of 1 indicate good agreement between the sensor values and NMM measurements.

\section{Results and Discussion}

\subsection{Vertical Soil Water Dynamics and Water Storage in the Soil Profile Using Neutron Moisture Meters}

Volumetric SWC measured by the NMMs ranged from 0.11 to $0.31 \mathrm{~m}^{3} \mathrm{~m}^{-3}$ at the $10-\mathrm{cm}$ depth during the corn growing season under three irrigation treatments (Figure 3). Conventional tillage in this study increased soil evaporation potential and deepened the evaporative drying front to at least $10 \mathrm{~cm}$, which resulted in large variation in SWC near the soil surface under irrigation conditions [34]. 
This is expected for a study being conducted in a high ET demand environment. The average annual standardized alfalfa reference ET (ETrs) is $\sim 1600 \mathrm{~mm}$ within the region [25]. The three irrigation treatments captured the wet and dry soil conditions given the PWP and FC of 0.18 and $0.33 \mathrm{~m}^{3} \mathrm{~m}^{-3}$, respectively (Figure 3). It was expected that the 100\% ETc treatment demonstrated relatively high SWC compared to the $75 \%$ and 50\% ETc treatments. From depths 30 to $130 \mathrm{~cm}$, the SWC varied from 0.24 to $0.38 \mathrm{~m}^{3} \mathrm{~m}^{-3}$. Smaller variations of 0.24 to $0.33 \mathrm{~m}^{3} \mathrm{~m}^{-3}$ in SWC were observed below the 150-cm depth for the Pullman clay loam soil (Figure 3). Integrated water storage in the $0.9 \mathrm{~m}$ soil profiles varied from 229 to $312 \mathrm{~mm}, 207$ to $310 \mathrm{~mm}$, and 207 to $308 \mathrm{~mm}$ with average values of 280, 261, and $243 \mathrm{~mm}$, respectively, under the $100 \%, 75 \%$, and $50 \%$ irrigation treatments (Table 1 ).
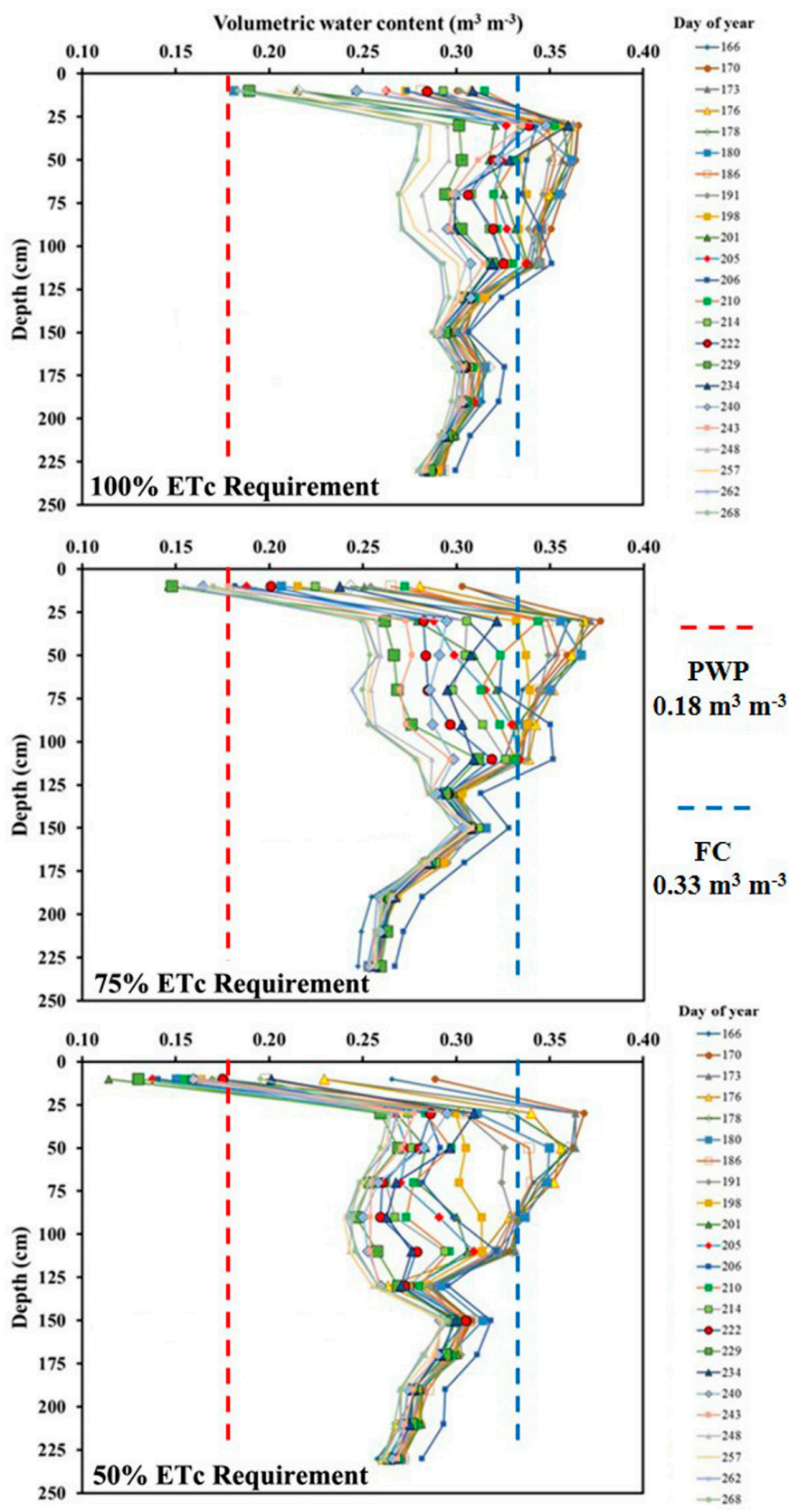

Figure 3. Volumetric soil water content $\left(\mathrm{m}^{3} \mathrm{~m}^{-3}\right)$ at the depths of 10, 30, 50, 70, 90, 110, 130, 150, 170, 190,210 , and $230 \mathrm{~cm}$ using the neutron moisture meters for days during the corn growing season under $100 \%, 75 \%$, and $50 \%$ crop evapotranspiration (ETc) requirements. 
Table 1. Water storage in the $0.9 \mathrm{~m}$ soil profiles $(\mathrm{mm})$ using the neutron moisture meters for three irrigation treatments.

\begin{tabular}{ccccc}
\hline Day of Year (DOY) & Date & $\mathbf{1 0 0 \%}$ ETc Treatment & $\mathbf{7 5 \%}$ ETc Treatment & 50\% ETc Treatment \\
\hline 166 & $6 / 15 / 2018$ & 307.1 & 303.5 & 299.7 \\
170 & $6 / 19 / 2018$ & 312.4 & 310.2 & 307.7 \\
173 & $6 / 22 / 2018$ & 297.3 & 299.7 & 294.0 \\
176 & $6 / 25 / 2018$ & 310.7 & 306.7 & 288.4 \\
178 & $6 / 27 / 2018$ & 291.5 & 298.0 & 275.1 \\
180 & $6 / 29 / 2018$ & 282.3 & 289.3 & 265.7 \\
186 & $7 / 5 / 2018$ & 300.8 & 296.4 & 269.3 \\
191 & $7 / 10 / 2018$ & 306.1 & 292.6 & 257.7 \\
198 & $7 / 17 / 2018$ & 293.4 & 278.4 & 245.3 \\
201 & $7 / 20 / 2018$ & 268.9 & 244.2 & 219.0 \\
205 & $7 / 24 / 2018$ & 279.5 & 250.9 & 218.8 \\
206 & $7 / 25 / 2018$ & 292.4 & 262.1 & 229.6 \\
210 & $7 / 29 / 2018$ & 296.0 & 282.8 & 229.8 \\
214 & $8 / 2 / 2018$ & 283.3 & 257.8 & 221.0 \\
222 & $8 / 10 / 2018$ & 281.8 & 239.8 & 226.3 \\
229 & $8 / 17 / 2018$ & 247.9 & 216.5 & 207.2 \\
234 & $8 / 22 / 2018$ & 289.2 & 262.6 & 241.3 \\
240 & $8 / 28 / 2018$ & 273.0 & 235.9 & 224.1 \\
243 & $8 / 31 / 2018$ & 271.2 & 226.7 & 218.7 \\
248 & $9 / 5 / 2018$ & 246.4 & 212.3 & 212.7 \\
257 & $9 / 14 / 2018$ & 237.4 & 211.3 & 212.9 \\
262 & $9 / 19 / 2018$ & 229.9 & 206.8 & 212.5 \\
268 & $9 / 25 / 2018$ & 229.4 & 209.8 & 217.8 \\
\hline Minimum value & 229.4 & 206.8 & 207.2 \\
Maximum value & 312.4 & 310.2 & 307.7 \\
Average value & 279.5 & 260.6 & 243.2 \\
\hline
\end{tabular}

\subsection{Soil Water Sensor Performance Using Factory Calibration}

Factory calibrated daily SWC values for each sensor type, depth, and installation orientation were calculated and compared with NMM measurements from similar depths during the corn growing season. In general, soil water sensor measurements, irrespective of installation orientation and irrigation treatment, were significantly different from the NMM measurements $(p<0.05)$ with relatively high SD values $>2.45 \%$ at the $15.2 \mathrm{~cm}$ depth, with the exception of the WM-200SS sensor (Table 2). Varble and Chávez [21] also found that the factory calibrated Acclima TDT, Decagon 5TE, and Campbell Scientific 616/625 sensors performed unsatisfactorily at a depth of $10 \mathrm{~cm}$ when compared to periodic thermogravimetrically determined SWC in a sandy clay loam soil in Colorado. According to Hignett and Evett [8], statistical goals of MD within $\pm 2 \%$ and RMSE $<3.5 \%$ for sensor applications in agricultural production and research are acceptable. Recently, Datta et al. [35] reported that the ACC-315L, DEC-GS1, and CS-655 sensors with factory calibration performed unsatisfactorily at the $20 \mathrm{~cm}$ depth when compared to thermogravimetric SWC measurements in a soil with a high level of clay content in Oklahoma. Differences between the NMM and soil water sensor measurements at the $15.2 \mathrm{~cm}$ depth were also observed, likely because the NMM does not typically perform well for measurement depths near the surface layer $[26,27]$. In addition, voids at the near surface layer can affect the sensor measurements.

In general, the ACC-315L sensor performed satisfactorily using the horizontal insertion with the factory calibration as compared to NMM measurements at the 45.7 and $76.2 \mathrm{~cm}$ depths for all three irrigation treatments, according to the Hignett and Evett [8] statistical goals (Tables 3 and 4). The ACC-315L sensors also captured most of the soil water variation using the horizontal insertion orientation $\left(R^{2}>0.73\right)$ at the 45.7 and $76.2 \mathrm{~cm}$ depths, with relatively small SD values $<3.21 \%$. The ACC-315L displayed the regression slopes that approximated the 1:1 line, with a range of 0.97 to 
1.61 under the three irrigation treatments. In general, the factory-calibrated DEC-GS1 sensor using vertical insertion performed better than other installation orientations for the depths of 45.7 and $76.2 \mathrm{~cm}$ according to the MD and RMSE values. However, no consistent trend regarding installation orientation was determined for the three irrigation treatments in the comparisons of the DEC-GS1 sensor with the corresponding NMM measurements. As such, field corrections are warranted for use in agricultural applications. Generally, the CS-655 sensor using vertical insertion outperformed other installation orientations at the 45.7 and $76.2 \mathrm{~cm}$ depths for $100 \%$ (wet) and 50\% (dry) irrigation treatments (Tables 3 and 4). However, further corrections were needed when using the CS-655 sensor for agricultural and research applications [8]. Significant MD $(p<0.05)$ and large RMSE were observed for the WM-200SS sensors at the depths of 45.7 and $76.2 \mathrm{~cm}$ with the three irrigation treatments using the factory calibration irrespective of installation orientation (Tables 3 and 4).

Table 2. Statistical summary of sensor performance at the $15.2 \mathrm{~cm}$ depth.

\begin{tabular}{|c|c|c|c|c|c|c|c|}
\hline $\begin{array}{c}\text { Sensor } \\
\text { (Treatment) }\end{array}$ & $\begin{array}{l}\text { Installation } \\
\text { Orientation }\end{array}$ & MD (\%) & RMSE (\%) & SD (\%) & Slope & Intercept & $R^{2}$ \\
\hline & \multicolumn{7}{|c|}{ Acclima 315L $(n=23)$} \\
\hline \multirow{3}{*}{$100 \%$ ETc } & Horizontal insertion & $7.51^{\mathrm{a}}$ & 9.43 & 5.69 & $0.68^{\mathrm{b}}$ & $15.62^{c}$ & 0.23 \\
\hline & Laid horizontal & $-7.37^{a}$ & 8.66 & 4.54 & $0.43^{\mathrm{b}}$ & 7.06 & 0.20 \\
\hline & Vertical insertion & $-6.45^{\mathrm{a}}$ & 9.26 & 6.65 & $1.26^{\mathrm{b}}$ & -12.99 & 0.42 \\
\hline \multirow{3}{*}{$75 \%$ ETc } & Horizontal insertion & $5.84^{\mathrm{a}}$ & 6.84 & 3.56 & $0.62^{b}$ & $14.04^{\mathrm{c}}$ & 0.50 \\
\hline & Laid horizontal & -1.66 & 3.47 & 3.05 & $0.62^{b}$ & $6.53^{c}$ & 0.62 \\
\hline & Vertical insertion & $-4.47^{\mathrm{a}}$ & 5.82 & 3.72 & $0.73^{b}$ & 1.23 & 0.52 \\
\hline \multirow{4}{*}{$50 \%$ ETc } & Horizontal insertion & $-2.32^{a}$ & 4.79 & 4.19 & 0.32 & $9.70^{c}$ & 0.16 \\
\hline & Laid horizontal & $-3.50^{\mathrm{a}}$ & 4.26 & 2.45 & $0.51^{\mathrm{b}}$ & $5.21^{\mathrm{c}}$ & 0.70 \\
\hline & Vertical insertion & $-4.97^{a}$ & 5.87 & 3.11 & $0.74^{\mathrm{b}}$ & -0.27 & 0.52 \\
\hline & \multicolumn{7}{|c|}{ Decagon GS1 $(n=23)$} \\
\hline \multirow{3}{*}{$100 \%$ ETc } & Horizontal insertion & $6.29^{a}$ & 8.25 & 5.33 & $1.49^{\mathrm{b}}$ & -6.21 & 0.65 \\
\hline & Laid horizontal & $6.66^{\mathrm{a}}$ & 7.65 & 3.76 & $1.07^{\mathrm{b}}$ & 4.96 & 0.61 \\
\hline & Vertical insertion & $7.31^{\mathrm{a}}$ & 8.22 & 3.78 & $0.73^{b}$ & $14.13^{c}$ & 0.45 \\
\hline \multirow{3}{*}{$75 \%$ ETc } & Horizontal insertion & $3.63^{\mathrm{a}}$ & 4.85 & 3.21 & $0.60^{\mathrm{b}}$ & $12.17^{\mathrm{c}}$ & 0.58 \\
\hline & Laid horizontal & 2.57 & 3.80 & 2.79 & $1.03^{\mathrm{b}}$ & 1.89 & 0.77 \\
\hline & Vertical insertion & $12.68^{\mathrm{a}}$ & 13.04 & 3.04 & $0.54^{\mathrm{b}}$ & $22.45^{c}$ & 0.63 \\
\hline \multirow{3}{*}{$50 \%$ ETc } & Horizontal insertion & $7.51^{\mathrm{a}}$ & 8.00 & 2.74 & $0.75^{\mathrm{b}}$ & $11.92^{c}$ & 0.60 \\
\hline & Laid horizontal & $-2.47^{\mathrm{a}}$ & 4.93 & 4.26 & $1.05^{\mathrm{b}}$ & -3.29 & 0.51 \\
\hline & Vertical insertion & $7.35^{\mathrm{a}}$ & 8.14 & 3.50 & $1.04^{\mathrm{b}}$ & 6.72 & 0.60 \\
\hline & \multicolumn{7}{|c|}{ Campbell Scientific 655} \\
\hline \multirow{3}{*}{$\begin{array}{c}100 \% \text { ETc } \\
(\mathrm{n}=23)\end{array}$} & Horizontal insertion & $13.71^{\mathrm{a}}$ & 14.43 & 4.49 & $0.56^{\mathrm{b}}$ & $24.84^{c}$ & 0.27 \\
\hline & Laid horizontal & $6.57^{\mathrm{a}}$ & 8.53 & 5.44 & $0.52^{b}$ & $18.85^{c}$ & 0.17 \\
\hline & Vertical insertion & $8.14^{\mathrm{a}}$ & 11.62 & 8.30 & $1.39^{b}$ & -1.70 & 0.36 \\
\hline \multirow{3}{*}{$\begin{array}{c}75 \% \text { ETc } \\
(n=23)\end{array}$} & Horizontal insertion & $7.26^{\mathrm{a}}$ & 8.53 & 4.48 & $1.10^{\mathrm{b}}$ & $5.07^{\mathrm{c}}$ & 0.60 \\
\hline & Laid horizontal & $-4.65^{\mathrm{a}}$ & 5.54 & 3.01 & $0.74^{\mathrm{b}}$ & 0.96 & 0.64 \\
\hline & Vertical insertion & $14.82^{\mathrm{a}}$ & 15.26 & 3.64 & $0.87^{b}$ & $17.59^{c}$ & 0.59 \\
\hline \multirow{4}{*}{$\begin{array}{l}50 \% \text { ETc } \\
(\mathrm{n}=17)\end{array}$} & Horizontal insertion & $11.85^{\mathrm{a}}$ & 12.28 & 3.25 & 0.37 & $22.08^{c}$ & 0.074 \\
\hline & Laid horizontal & 1.43 & 3.05 & 2.69 & 0.37 & $11.54^{\mathrm{c}}$ & 0.12 \\
\hline & Vertical insertion & $-2.20^{a}$ & 4.62 & 4.06 & 0.59 & 4.48 & 0.10 \\
\hline & \multicolumn{7}{|c|}{ Watermark 200SS } \\
\hline \multirow{3}{*}{$\begin{array}{c}100 \% \text { ETc } \\
(\mathrm{n}=23)\end{array}$} & Horizontal insertion & -1.58 & 4.19 & 3.88 & $0.50^{\mathrm{b}}$ & $11.20^{\mathrm{c}}$ & 0.33 \\
\hline & Laid horizontal & -2.28 & 3.78 & 3.01 & $0.54^{\mathrm{b}}$ & $9.29^{c}$ & 0.54 \\
\hline & Vertical insertion & -1.22 & 4.31 & 4.13 & $0.48^{\mathrm{b}}$ & $11.88^{c}$ & 0.28 \\
\hline \multirow{3}{*}{$\begin{array}{c}75 \% \text { ETc } \\
(n=23)\end{array}$} & Horizontal insertion & -1.97 & 3.97 & 3.44 & $0.41^{\mathrm{b}}$ & $10.73^{c}$ & 0.55 \\
\hline & Laid horizontal & -0.18 & 3.40 & 3.40 & $0.42^{b}$ & $12.15^{\mathrm{c}}$ & 0.56 \\
\hline & Vertical insertion & -1.52 & 3.65 & 3.32 & $0.44^{\mathrm{b}}$ & $10.54^{\mathrm{c}}$ & 0.58 \\
\hline
\end{tabular}


Table 2. Cont.

\begin{tabular}{cccccccc}
\hline \multirow{2}{*}{$50 \%$ ETc } & Horizontal insertion & -1.32 & 2.48 & 2.09 & 0.087 & $16.07^{\mathrm{c}}$ & 0.19 \\
$(\mathrm{n}=17)$ & Laid horizontal & 1.37 & 2.49 & 2.08 & 0.12 & $15.57^{\mathrm{c}}$ & 0.16 \\
& Vertical insertion & 1.08 & 2.41 & 2.15 & 0.05 & $16.42^{\mathrm{c}}$ & 0.18 \\
\hline
\end{tabular}

${ }^{\mathrm{a}} \mathrm{MD}$ (mean difference) is significantly different from zero at $p<0.05$ level. ${ }^{\mathrm{b}}$ Slope was significantly different from zero at $p<0.05$ level. ${ }^{\mathrm{c}}$ Intercept is significantly different from zero at $p<0.05$ level. RMSE indicates root mean square error. SD indicates standard deviation.

Table 3. Statistical summary of sensor performance at the $45.7 \mathrm{~cm}$ depth.

\begin{tabular}{|c|c|c|c|c|c|c|c|}
\hline $\begin{array}{c}\text { Sensor } \\
\text { (Treatment) }\end{array}$ & $\begin{array}{l}\text { Installation } \\
\text { Orientation }\end{array}$ & MD (\%) & RMSE (\%) & SD (\%) & Slope ${ }^{b}$ & Intercept & $R^{2}$ \\
\hline & \multicolumn{7}{|c|}{ Acclima 315L $(n=23)$} \\
\hline \multirow{3}{*}{$100 \%$ ETc } & Horizontal insertion & 2.04 & 3.17 & 2.42 & 1.36 & -9.92 & 0.73 \\
\hline & Laid horizontal & $-3.92^{\mathrm{a}}$ & 6.40 & 5.07 & 2.19 & $-43.18^{c}$ & 0.70 \\
\hline & Vertical insertion & $-9.35^{a}$ & 12.25 & 7.91 & 2.77 & $-67.68^{c}$ & 0.58 \\
\hline \multirow{3}{*}{$75 \%$ ETc } & Horizontal insertion & -0.75 & 2.36 & 2.23 & 1.09 & -3.61 & 0.79 \\
\hline & Laid horizontal & $-8.44^{\mathrm{a}}$ & 9.89 & 5.16 & 1.22 & -15.44 & 0.47 \\
\hline & Vertical insertion & $-12.51^{\mathrm{a}}$ & 14.31 & 6.95 & 2.08 & $-46.27^{c}$ & 0.69 \\
\hline \multirow{4}{*}{$50 \%$ ETc } & Horizontal insertion & 0.088 & 2.04 & 2.04 & 1.28 & $-8.55^{c}$ & 0.89 \\
\hline & Laid horizontal & $-9.76^{\mathrm{a}}$ & 10.38 & 3.54 & 0.77 & -2.80 & 0.42 \\
\hline & Vertical insertion & $-16.17^{a}$ & 17.48 & 6.62 & 2.35 & $-57.34^{c}$ & 0.82 \\
\hline & \multicolumn{7}{|c|}{ Decagon GS1 $(n=23)$} \\
\hline \multirow{3}{*}{$100 \%$ ETc } & Horizontal insertion & -0.50 & 5.79 & 5.77 & 2.73 & $-57.50^{c}$ & 0.83 \\
\hline & Laid horizontal & -2.74 & 6.87 & 6.30 & 2.52 & $-52.69^{c}$ & 0.67 \\
\hline & Vertical insertion & $5.03^{\mathrm{a}}$ & 5.57 & 2.38 & 1.31 & -5.10 & 0.71 \\
\hline \multirow{3}{*}{$75 \%$ ETc } & Horizontal insertion & $3.19^{\mathrm{a}}$ & 3.84 & 2.14 & 1.01 & 2.79 & 0.78 \\
\hline & Laid horizontal & $-3.84^{\mathrm{a}}$ & 6.08 & 4.71 & 1.80 & $-28.80^{c}$ & 0.80 \\
\hline & Vertical insertion & -3.77 & 4.82 & 3.02 & 1.35 & $-14.67^{c}$ & 0.80 \\
\hline \multirow{4}{*}{$50 \%$ ETc } & Horizontal insertion & -0.21 & 5.06 & 5.05 & 1.67 & $-20.56^{c}$ & 0.68 \\
\hline & Laid horizontal & $-8.31^{\mathrm{a}}$ & 8.59 & 2.16 & 1.08 & $-10.65^{c}$ & 0.78 \\
\hline & Vertical insertion & -0.58 & 3.08 & 3.02 & 1.42 & $-13.27^{c}$ & 0.81 \\
\hline & \multicolumn{7}{|c|}{ Campbell Scientific 655} \\
\hline \multirow{3}{*}{$\begin{array}{c}100 \% \text { ETc } \\
(\mathrm{n}=23)\end{array}$} & Horizontal insertion & $8.57^{\mathrm{a}}$ & 9.03 & 2.86 & 1.65 & -12.70 & 0.79 \\
\hline & Laid horizontal & 0.62 & 5.03 & 4.99 & 2.34 & $-43.45^{c}$ & 0.77 \\
\hline & Vertical insertion & 2.90 & 9.36 & 8.90 & 3.93 & $-93.39^{c}$ & 0.87 \\
\hline \multirow{3}{*}{$\begin{array}{c}75 \% \text { ETc } \\
(n=23)\end{array}$} & Horizontal insertion & $6.54^{\mathrm{a}}$ & 6.89 & 2.16 & 1.09 & 3.76 & 0.80 \\
\hline & Laid horizontal & $-15.49^{a}$ & 15.77 & 2.95 & 0.96 & $-14.18^{c}$ & 0.62 \\
\hline & Vertical insertion & $-5.76^{\mathrm{a}}$ & 9.62 & 7.71 & 2.30 & $-46.69^{c}$ & 0.71 \\
\hline \multirow{4}{*}{$\begin{array}{c}50 \% \text { ETc } \\
(\mathrm{n}=17)\end{array}$} & Horizontal insertion & $9.61^{a}$ & 9.72 & 1.42 & 0.39 & $27.03^{c}$ & 0.77 \\
\hline & Laid horizontal & $-13.08^{a}$ & 13.31 & 2.43 & 1.43 & $-25.40^{c}$ & 0.66 \\
\hline & Vertical insertion & -0.94 & 1.82 & 1.56 & 0.32 & $18.37^{c}$ & 0.69 \\
\hline & \multicolumn{7}{|c|}{ Watermark 200SS } \\
\hline \multirow{3}{*}{$\begin{array}{c}100 \% \text { ETc } \\
(\mathrm{n}=23)\end{array}$} & Horizontal insertion & $-8.69^{a}$ & 9.05 & 2.56 & 1.29 & $-18.22^{c}$ & 0.67 \\
\hline & Laid horizontal & $-9.16^{a}$ & 10.06 & 4.16 & 1.52 & $-26.39^{c}$ & 0.53 \\
\hline & Vertical insertion & $-10.01^{\mathrm{a}}$ & 10.49 & 3.11 & 1.52 & $-27.12^{c}$ & 0.68 \\
\hline \multirow{3}{*}{$\begin{array}{c}75 \% \text { ETc } \\
(\mathrm{n}=23)\end{array}$} & Horizontal insertion & $-11.01^{a}$ & 11.22 & 2.16 & 0.73 & -2.57 & 0.70 \\
\hline & Laid horizontal & $-11.85^{\mathrm{a}}$ & 12.08 & 2.34 & 0.64 & -0.48 & 0.65 \\
\hline & Vertical insertion & $-11.67^{\mathrm{a}}$ & 11.92 & 2.41 & 0.74 & -3.35 & 0.64 \\
\hline \multirow{3}{*}{$\begin{array}{c}50 \% \text { ETc } \\
(\mathrm{n}=17)\end{array}$} & Horizontal insertion & $-11.10^{a}$ & 11.24 & 1.75 & 0.22 & $11.15^{c}$ & 0.65 \\
\hline & Laid horizontal & $-11.26^{a}$ & 11.41 & 1.81 & 0.19 & $11.89^{c}$ & 0.60 \\
\hline & Vertical insertion & $-11.13^{a}$ & 11.28 & 1.81 & 0.18 & $12.18^{c}$ & 0.67 \\
\hline
\end{tabular}

${ }^{a} \mathrm{MD}$ (mean difference) is significantly different from zero at $p<0.05$ level. ${ }^{\mathrm{b}}$ Slope was significantly different from zero at $p<0.05$ level. ${ }^{c}$ Intercept is significantly different from zero at $p<0.05$ level. RMSE indicates root mean square error. SD indicates standard deviation. 
Table 4. Statistical summary of sensor performance at the $76.2 \mathrm{~cm}$ depth.

\begin{tabular}{|c|c|c|c|c|c|c|c|}
\hline $\begin{array}{c}\text { Sensor } \\
\text { (Treatment) }\end{array}$ & $\begin{array}{l}\text { Installation } \\
\text { orientation }\end{array}$ & MD (\%) & RMSE (\%) & $\mathrm{SD}(\%)$ & Slope $^{b}$ & Intercept & $R^{2}$ \\
\hline & \multicolumn{7}{|c|}{ Acclima 315L $(n=23)$} \\
\hline \multirow{3}{*}{$100 \%$ ETc } & Horizontal insertion & 2.02 & 2.60 & 1.64 & 1.47 & $-12.84^{\mathrm{c}}$ & 0.96 \\
\hline & Laid horizontal & $-6.85^{a}$ & 8.28 & 4.65 & 2.06 & $-40.68^{c}$ & 0.75 \\
\hline & Vertical insertion & $-9.12^{\mathrm{a}}$ & 11.14 & 6.39 & 2.52 & $-57.56^{c}$ & 0.72 \\
\hline \multirow{3}{*}{$75 \%$ ETc } & Horizontal insertion & 0.42 & 1.87 & 1.83 & 0.97 & 1.31 & 0.79 \\
\hline & Laid horizontal & $-11.73^{\mathrm{a}}$ & 12.14 & 3.13 & 1.15 & $-16.40^{\mathrm{c}}$ & 0.65 \\
\hline & Vertical insertion & $-9.57^{a}$ & 10.74 & 4.87 & 1.47 & $-23.90^{c}$ & 0.58 \\
\hline \multirow{4}{*}{$50 \%$ ETc } & Horizontal insertion & -1.52 & 3.55 & 3.21 & 1.61 & $-19.29^{c}$ & 0.90 \\
\hline & Laid horizontal & $-13.38^{a}$ & 13.60 & 2.47 & 0.53 & 0.36 & 0.63 \\
\hline & Vertical insertion & $-8.83^{\mathrm{a}}$ & 9.48 & 3.45 & 1.68 & $-28.57^{\mathrm{c}}$ & 0.90 \\
\hline & \multicolumn{7}{|c|}{ Decagon GS1 $(n=23)$} \\
\hline \multirow{3}{*}{$100 \%$ ETc } & Horizontal insertion & $-4.78^{a}$ & 8.46 & 6.97 & 2.76 & $-61.14^{c}$ & 0.75 \\
\hline & Laid horizontal & -4.03 & 7.95 & 6.85 & 2.79 & $-61.10^{c}$ & 0.78 \\
\hline & Vertical insertion & 3.60 & 4.39 & 2.51 & 1.40 & -9.28 & 0.78 \\
\hline \multirow{3}{*}{$75 \%$ ETc } & Horizontal insertion & $2.71^{\mathrm{a}}$ & 3.27 & 1.83 & 1.01 & 2.43 & 0.80 \\
\hline & Laid horizontal & $-16.67^{a}$ & 17.55 & 5.48 & 1.82 & $-41.85^{c}$ & 0.67 \\
\hline & Vertical insertion & $-6.60^{a}$ & 8.25 & 4.95 & 1.61 & $-25.43^{c}$ & 0.64 \\
\hline \multirow{4}{*}{$50 \%$ ETc } & Horizontal insertion & $4.33^{\mathrm{a}}$ & 4.43 & 0.98 & 1.10 & 1.52 & 0.96 \\
\hline & Laid horizontal & $-13.50^{a}$ & 14.10 & 4.08 & 1.62 & $-31.47^{c}$ & 0.80 \\
\hline & Vertical insertion & 2.84 & 3.25 & 1.57 & 1.24 & -4.09 & 0.94 \\
\hline & \multicolumn{7}{|c|}{ Campbell Scientific 655} \\
\hline \multirow{3}{*}{$\begin{array}{c}100 \% \text { ETc } \\
(n=23)\end{array}$} & Horizontal insertion & $10.77^{a}$ & 10.94 & 1.94 & 1.33 & 0.31 & 0.84 \\
\hline & Laid horizontal & 3.13 & 6.92 & 6.17 & 2.44 & $-42.99^{c}$ & 0.72 \\
\hline & Vertical insertion & 0.35 & 8.62 & 8.61 & 3.71 & $-86.94^{c}$ & 0.92 \\
\hline \multirow{3}{*}{$\begin{array}{c}75 \% \text { ETc } \\
(n=23)\end{array}$} & Horizontal insertion & $3.88^{\mathrm{a}}$ & 4.95 & 3.08 & 1.28 & -4.57 & 0.72 \\
\hline & Laid horizontal & $-14.77^{a}$ & 16.85 & 8.11 & 2.09 & $-48.14^{\mathrm{c}}$ & 0.53 \\
\hline & Vertical insertion & $-15.99^{a}$ & 17.11 & 6.09 & 1.87 & $-42.60^{\mathrm{c}}$ & 0.63 \\
\hline \multirow{4}{*}{$\begin{array}{c}50 \% \text { ETc } \\
(n=17)\end{array}$} & Horizontal insertion & $8.32^{a}$ & 8.40 & 1.06 & 0.72 & $15.86^{c}$ & 0.85 \\
\hline & Laid horizontal & $-11.69^{a}$ & 11.75 & 1.25 & 0.66 & -2.35 & 0.79 \\
\hline & Vertical insertion & -0.37 & 0.93 & 0.86 & 1.43 & $-11.70^{\mathrm{c}}$ & 0.92 \\
\hline & \multicolumn{7}{|c|}{ Watermark 200SS } \\
\hline \multirow{3}{*}{$\begin{array}{c}100 \% \text { ETc } \\
(n=23)\end{array}$} & Horizontal insertion & $-7.06^{\mathrm{a}}$ & 7.38 & 2.16 & 1.19 & $-13.02^{\mathrm{c}}$ & 0.74 \\
\hline & Laid horizontal & $-7.85^{\mathrm{a}}$ & 8.36 & 2.88 & 1.33 & $-18.31^{\mathrm{c}}$ & 0.67 \\
\hline & Vertical insertion & $-8.29^{a}$ & 9.13 & 3.84 & 1.41 & $-21.36^{c}$ & 0.56 \\
\hline \multirow{3}{*}{$\begin{array}{l}75 \% \text { ETc } \\
(n=23)\end{array}$} & Horizontal insertion & $-9.79^{a}$ & 10.01 & 2.09 & 0.77 & -2.60 & 0.68 \\
\hline & Laid horizontal & $-10.12^{a}$ & 10.33 & 2.10 & 0.85 & -5.61 & 0.70 \\
\hline & Vertical insertion & $-10.32^{a}$ & 10.52 & 2.03 & 0.84 & -5.43 & 0.71 \\
\hline \multirow{3}{*}{$\begin{array}{c}50 \% \text { ETc } \\
(n=17)\end{array}$} & Horizontal insertion & $-9.54^{\mathrm{a}}$ & 9.68 & 1.64 & 0.41 & $6.67^{c}$ & 0.77 \\
\hline & Laid horizontal & $-9.91^{\mathrm{a}}$ & 10.10 & 1.94 & 0.27 & $9.96^{c}$ & 0.71 \\
\hline & Vertical insertion & $-9.81^{\mathrm{a}}$ & 9.97 & 1.77 & 0.36 & $7.57^{\mathrm{c}}$ & 0.70 \\
\hline
\end{tabular}

\footnotetext{
${ }^{\mathrm{a}} \mathrm{MD}$ (mean difference) is significantly different from zero at $p<0.05$ level. ${ }^{\mathrm{b}}$ Slope was significantly different from zero at $p<0.05$ level. ${ }^{\mathrm{c}}$ Intercept is significantly different from zero at $p<0.05$ level. RMSE indicates root mean square error. SD indicates standard deviation.
}

\subsection{Comparison of Integrated Soil Profile Water Storage Using Factory Calibration under Three Irrigation Treatments}

Comparison of water storage in the $0.9 \mathrm{~m}$ soil profile integrated from both the soil water sensors and NMM measurements indicated the factory-calibrated ACC-315L sensors performed satisfactorily using the horizontal insertion orientation under the $75 \%$ ETc treatment $(\mathrm{RMSE}=20.88 \mathrm{~mm}$ and $2.32 \%$ 
and $\mathrm{MD}=6.50 \mathrm{~mm}$ or $0.72 \%$ ), according to the Hignett and Evett [8] statistical goals (Figure 4). In addition, the DEC-GS1 sensor using vertical insertion did not require correction under the $75 \%$ and $50 \%$ ETc treatments. The MD, RMSE, $R^{2}$, and slope were $-0.34 \%$ and $1.96 \%, 2.89 \%$ and $3.05 \%$, 0.75 and 0.92 , and 1.23 and 1.50 for the vertically inserted DEC-GS1 sensors under the $75 \%$ and $50 \%$ ETc treatments, respectively (Figures 4 and 5). The 75\% irrigation treatment represents the dominant "deficit irrigation strategy" used with limited well capacities in the Texas High Plains. This management level is necessitated by the reduced well capacity as well as regional groundwater district regulation (pumping limits). The satisfactory performance of the factory-calibrated ACC-315L and DEC-GS1 sensors for specific installation orientations for the $75 \%$ ETc treatment may provide useful information for certain producers, consultants, and extension specialists. Similar to the CS-655 sensor comparison at depths, the associated soil profile water storage values integrated from CS-655 sensors using vertical insertion outperformed other installation orientations but needed correction for agricultural applications under all three irrigation treatments (Figure S2, Figures 4 and 5). Again, significant MD and large RMSE were found with the WM-200SS sensors. The WM-200SS sensors significantly underestimated water storage within the $0.9 \mathrm{~m}$ soil profile when compared to the NMM values under the three irrigation treatments (Figures S2, Figures 4 and 5).
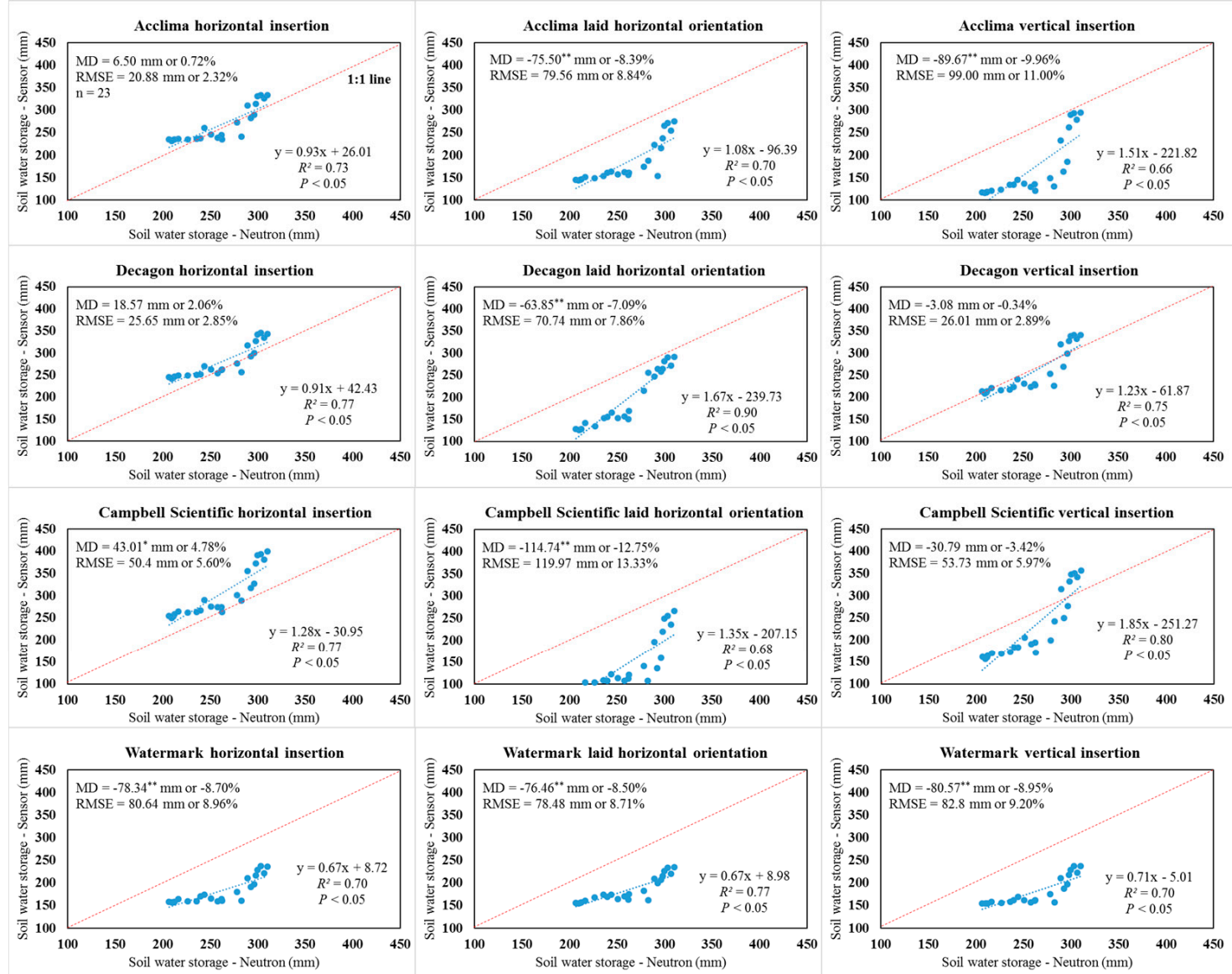

Figure 4. Graphical and statistical comparisons of sensor and neutron moisture meter-derived soil profile water storage values in the upper $0.9 \mathrm{~m}$ soil profile under the $75 \%$ crop evapotranspiration treatment. MD indicates mean difference; RMSE indicates root mean square error; ${ }^{*}$ indicates a significant difference at $p<0.05 ;{ }^{* *}$ indicates a significant difference at $p<0.01$. 


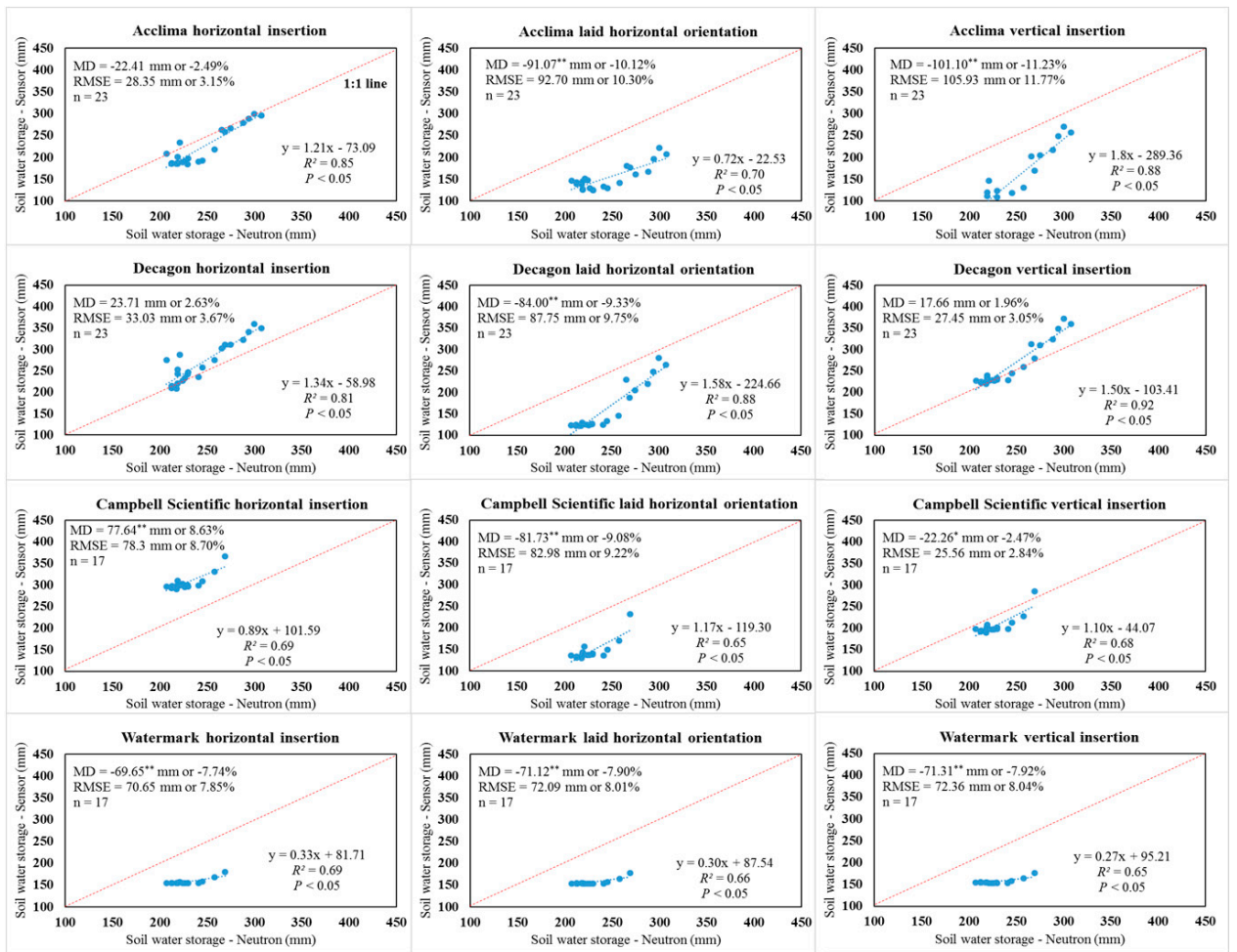

Figure 5. Graphical and statistical comparisons of sensor and neutron moisture meter-derived soil profile water storage values in the upper $0.9 \mathrm{~m}$ soil profile under the $50 \%$ crop evapotranspiration treatment. MD indicates mean difference; RMSE indicates root mean square error; ${ }^{*}$ indicates a significant difference at $p<0.05 ;{ }^{* *}$ indicates a significant difference at $p<0.01$.

As shown in Figure 6, pooled data $(n=69)$ from all irrigation treatments for the ACC-315L sensors using horizontal insertion were acceptable for use in agricultural production and research settings using the factory calibration. In view of the MD of soil water storage in the profile, the DEC-GS1 sensors using the horizontal and vertical insertions might also be used without correction (Figure 6). However, the MD of the sensor comparison of individual depths indicated corrections were required for the DEC-GS1 sensor for agricultural applications in all three irrigation treatments. Therefore, the Hignett and Evett [8] statistical goals of "MD within $\pm 2 \%$ and RMSE $<3.5 \%$ " were more suitable for a specific depth or point comparison. As for comparisons for profile water storage values, the statistical goals of "MD within $\pm 1 \%$ and RMSE $<3.5 \%$ " were proposed using soil water sensors in irrigation scheduling and related agricultural applications. Therefore, DEC-GS1 sensors required corrections based on the proposed statistical goals. Results showed readings from the ACC-315L sensor were also sensitive to installation orientation. Soil water storage was significantly underestimated by ACC-315L sensors using both the laid horizontal and vertical insertion orientations under all three irrigation treatments (Figure 6). Measurements from the TDR-based ACC-315L should not be affected by sensor orientation. However, differences in soil bulk density attributed to the manually packed (laid horizontal) and undisturbed (horizontal and vertical) insertion likely influenced measurements. In general, the soil was less distributed under the horizontal insertion as compared to the laid horizontal orientation, resulting in higher bulk densities and reasonable agreement with NMM readings (Figure 4). The differences associated with horizontal and vertical insertion orientations are more difficult to explain as they were also installed in undisturbed soil. It is uncertain if measurements were affected by a vertical soil temperature gradient, as temperature was not integrated with depth, but was measured at a single point by a singular thermistor located just inside the sensor head in the outer waveguide. The ACC-315L integrated water content along the length of the probe, resulting in a mean water content. These may partially explain the difference in values associated with the installation 
orientations, particularly for the sensors positioned at the $15.2 \mathrm{~cm}$ depth as a considerable SWC gradient may exist near the soil surface following soil wetting events. The CS- 655 sensor tended to overestimate the profile water storage under relatively wet conditions $(280-330 \mathrm{~mm})$ with deviations from the 1:1 line being large, with slopes higher than two using the laid horizontal and vertical insertion orientations. Even though significant MD was identified for the WM-200SS sensors, the slopes were all close to one, which indicated a systemic underestimation of the soil water storage when using the WM-200SS sensors, regardless of the installation orientation and irrigation treatment (Figure 6).

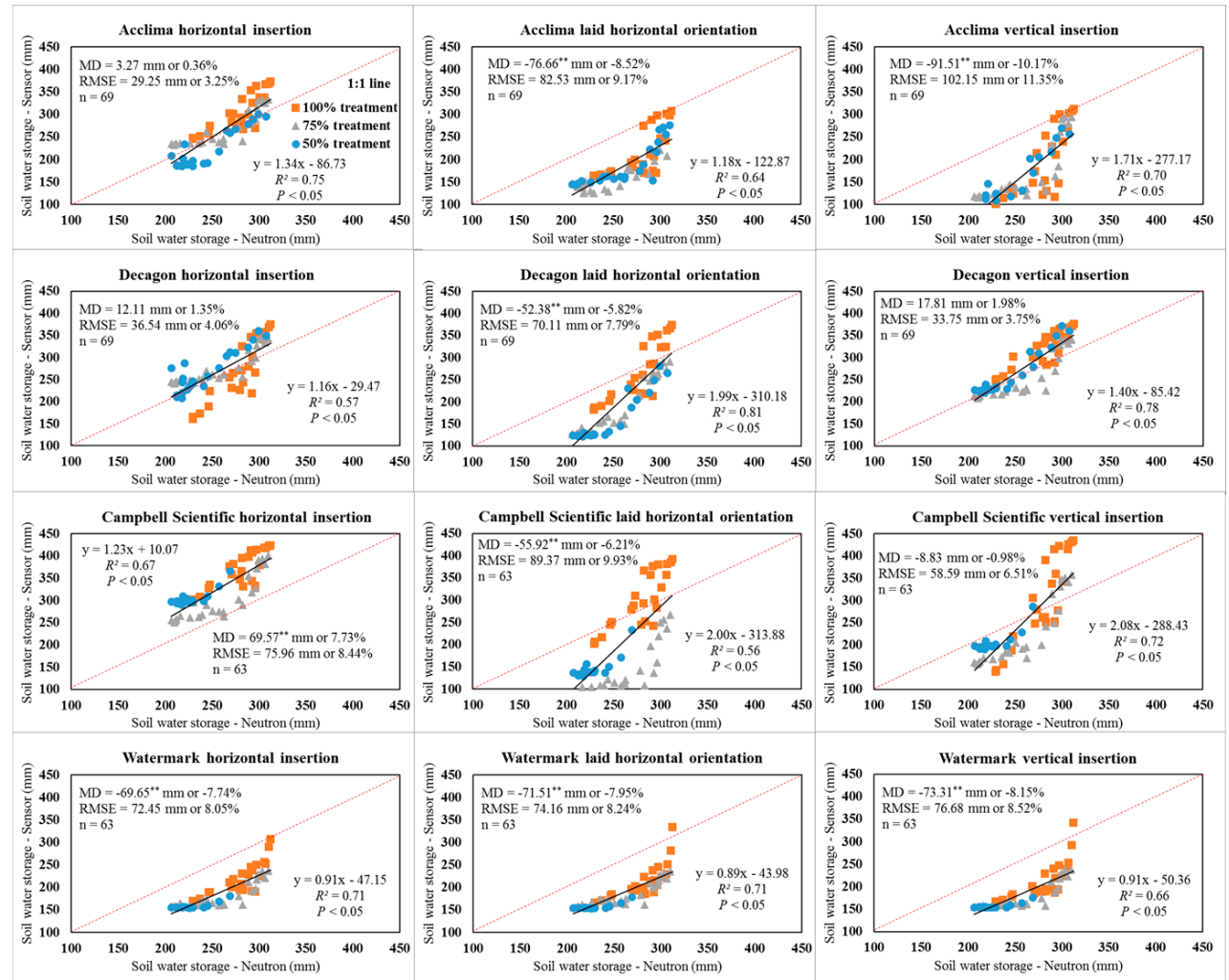

Figure 6. Graphical and statistical comparisons of sensor and neutron moisture meter-derived soil profile water storage values in the upper $0.9 \mathrm{~m}$ soil profile when combining all the data from three irrigation treatments. MD indicates mean difference; RMSE indicates root mean square error; ** indicates a significant difference at $p<0.01$.

\subsection{Field Corrections of Integrated Soil Profile Water Storage}

Results showed that all soil water sensors could be used for irrigation scheduling in this study region when used with a field correction based on the proposed statistical goals. As for the field correction equations for soil profile water storage, the quadratic equation had the highest $R^{2}$ for the four sensors (Table 5). It is worth noting that all field correction equations used in this study for the DEC-GS1 sensors using the horizontal insertion resulted in an $R^{2}<0.65$, which generally would not be deemed acceptable. Therefore, in addition to the MD and RMSE, we recommend the adopted fitting equation to have an $R^{2}>0.65$ for irrigation scheduling purposes and associated applications. The suggested evaluation criteria for soil water sensor performance, therefore, is MD within $\pm 1 \%$, RMSE $<3.5 \%$, and $R^{2}>0.65$ for data fitting using soil profile water storage (Table 5). According to these evaluation criteria, the DEC-GS1 sensors using a vertical insertion may be appropriate for agricultural water management within this study region when using the field correction equations developed from this study data. It is also worth noting that the field-corrected CS-655 sensors in this study had larger $R^{2}$ values using the vertical insertion relative to those using horizontal insertion (Table 5). Assuming a 
vertical orientation represents a more integrated value in terms of the soil profile layers useful for irrigation management.

Table 5. Field correction equations developed for four soil water sensors in the Pullman clay loam soil at Bushland, TX, USA.

\begin{tabular}{|c|c|c|c|c|c|}
\hline Field Correction & Equation Type & Equation & MD (\%) & $\begin{array}{c}\text { RMSE } \\
(\%)\end{array}$ & $R^{2}$ \\
\hline \multirow{4}{*}{$\begin{array}{c}\text { Acclima } \\
\text { horizontal insertion } \\
(\mathrm{n}=69)\end{array}$} & Linear & \multirow{4}{*}{$\begin{array}{c}\theta_{\mathrm{v}}=0.56 \times \theta_{\mathrm{vi}}+12.69 \\
\theta_{\mathrm{v}}=16.31 \times \mathrm{e}^{0.019 \times \theta_{\mathrm{vi}}} \\
\theta_{\mathrm{v}}=16.12 \times \ln \left(\theta_{\mathrm{vi}}\right)-25.15 \\
\theta_{\mathrm{v}}=-0.0062 \times\left(\theta_{\mathrm{vi}}\right)^{2}+0.93 \\
\times \theta_{\mathrm{vi}}+7.31\end{array}$} & -0.00007 & 1.90 & 0.75 \\
\hline & Exponential & & -0.065 & 1.94 & 0.73 \\
\hline & Logarithmic & & 0.0017 & 1.91 & 0.75 \\
\hline & Quadratic & & 0.0026 & 1.89 & 0.75 \\
\hline \multirow{4}{*}{$\begin{array}{c}\text { Decagon } \\
\text { horizontal insertion } \\
(\mathrm{n}=69)\end{array}$} & Linear & \multirow{4}{*}{$\begin{array}{c}\theta_{\mathrm{v}}=0.49 \times \theta_{\mathrm{vi}}+14.02 \\
\theta_{\mathrm{v}}=17.19 \times \mathrm{e}^{0.017 \times \theta_{\mathrm{vi}}} \\
\theta_{\mathrm{v}}=14.12 \times \ln \left(\theta_{\mathrm{vi}}\right)-18.91 \\
\theta_{\mathrm{v}}=0.016 \times\left(\theta_{\mathrm{vi}}\right)^{2}-0.46 \times \\
\theta_{\mathrm{vi}}+28.22\end{array}$} & -0.00015 & 2.47 & 0.57 \\
\hline & Exponential & & -0.074 & 2.44 & 0.55 \\
\hline & Logarithmic & & -0.00048 & 2.58 & 0.53 \\
\hline & Quadratic & & 0.030 & 2.40 & 0.60 \\
\hline \multirow{4}{*}{$\begin{array}{l}\text { Decagon } \\
\text { vertical insertion } \\
\quad(n=69)\end{array}$} & Linear & \multirow{4}{*}{$\begin{array}{c}\theta_{\mathrm{v}}=0.56 \times \theta_{\mathrm{vi}}+11.71 \\
\theta_{\mathrm{v}}=15.76 \times \mathrm{e}^{0.019 \times \theta_{\mathrm{vi}}} \\
\theta_{\mathrm{v}}=17.58 \times \ln \left(\theta_{\mathrm{vi}}\right)-31.02 \\
\theta_{\mathrm{v}}=-0.018 \times\left(\theta_{\mathrm{vi}}\right)^{2}+1.72 \times \\
\theta_{\mathrm{vi}}-6.19\end{array}$} & 0.0011 & 1.78 & 0.78 \\
\hline & Exponential & & -0.063 & 1.82 & 0.76 \\
\hline & Logarithmic & & -0.00048 & 1.73 & 0.79 \\
\hline & Quadratic & & -0.048 & 1.72 & 0.79 \\
\hline \multirow{4}{*}{$\begin{array}{l}\text { Campbell Scientific } \\
\text { horizontal insertion } \\
\qquad(\mathrm{n}=63)\end{array}$} & Linear & \multirow{4}{*}{$\begin{array}{c}\theta_{\mathrm{v}}=0.55 \times \theta_{\mathrm{vi}}+8.84 \\
\theta_{\mathrm{v}}=14.27 \times \mathrm{e}^{0.019 \times \theta_{\mathrm{vi}}} \\
\theta_{\mathrm{v}}=20.11 \times \ln \left(\theta_{\mathrm{vi}}\right)-43.33 \\
\theta_{\mathrm{v}}=-0.0001 \times\left(\theta_{\mathrm{vi}}\right)^{2}+0.55 \\
\times \theta_{\mathrm{vi}}+8.69\end{array}$} & 0.0010 & 2.18 & 0.67 \\
\hline & Exponential & & -0.14 & 2.19 & 0.66 \\
\hline & Logarithmic & & 0.0015 & 2.19 & 0.67 \\
\hline & Quadratic & & 0.0072 & 2.18 & 0.67 \\
\hline \multirow{4}{*}{$\begin{array}{l}\text { Campbell Scientific } \\
\text { vertical insertion } \\
\qquad(\mathrm{n}=63)\end{array}$} & Linear & \multirow{4}{*}{$\begin{array}{c}\theta_{\mathrm{v}}=0.35 \times \theta_{\mathrm{vi}}+19.14 \\
\theta_{\mathrm{v}}=20.45 \times \mathrm{e}^{0.012 \times \theta_{\mathrm{vi}}} \\
\theta_{\mathrm{v}}=10.40 \times \ln \left(\theta_{\mathrm{vi}}\right)-5.30 \\
\theta_{\mathrm{v}}=-0.011 \times\left(\theta_{\mathrm{vi}}\right)^{2}+1.04 \times \\
\theta_{\mathrm{vi}}+9.25\end{array}$} & 0.00048 & 2.02 & 0.72 \\
\hline & Exponential & & -0.088 & 2.09 & 0.69 \\
\hline & Logarithmic & & -0.00060 & 1.90 & 0.75 \\
\hline & Quadratic & & 0.035 & 1.83 & 0.77 \\
\hline \multirow{4}{*}{$\begin{array}{c}\text { Watermark } \\
\text { horizontal insertion } \\
\qquad(\mathrm{n}=63)\end{array}$} & Linear & \multirow{4}{*}{$\begin{array}{c}\theta_{\mathrm{v}}=0.78 \times \theta_{\mathrm{vi}}+12.33 \\
\theta_{\mathrm{v}}=16.16 \times \mathrm{e}^{0.027 \times \theta_{\mathrm{vi}}} \\
\theta_{\mathrm{v}}=18.14 \times \ln \left(\theta_{\mathrm{vi}}\right)-26.18 \\
\theta_{\mathrm{v}}=-0.057 \times\left(\theta_{\mathrm{vi}}\right)^{2}+3.41 \times \\
\theta_{\mathrm{vi}}-17.03\end{array}$} & -0.00005 & 2.04 & 0.71 \\
\hline & Exponential & & -0.066 & 2.18 & 0.69 \\
\hline & Logarithmic & & -0.00004 & 1.87 & 0.76 \\
\hline & Quadratic & & -0.0039 & 1.70 & 0.80 \\
\hline \multirow{4}{*}{$\begin{array}{l}\text { Watermark laid } \\
\text { horizontal } \\
\text { orientation } \\
(n=63)\end{array}$} & Linear & \multirow{4}{*}{$\begin{array}{c}\theta_{\mathrm{v}}=0.80 \times \theta_{\mathrm{vi}}+12.11 \\
\theta_{\mathrm{v}}=16.04 \times \mathrm{e}^{0.028 \times \theta_{\mathrm{vi}}} \\
\theta_{\mathrm{v}}=19.07 \times \ln \left(\theta_{\mathrm{vi}}\right)-28.81 \\
\theta_{\mathrm{v}}=-0.054 \times\left(\theta_{\mathrm{vi}}\right)^{2}+3.37 \times \\
\theta_{\mathrm{vi}}-17.14\end{array}$} & -0.00051 & 2.03 & 0.71 \\
\hline & Exponential & & -0.071 & 2.28 & 0.69 \\
\hline & Logarithmic & & 0.0016 & 1.77 & 0.78 \\
\hline & Quadratic & & -0.014 & 1.46 & 0.85 \\
\hline \multirow{4}{*}{$\begin{array}{c}\text { Watermark } \\
\text { vertical insertion } \\
\qquad(n=63)\end{array}$} & Linear & \multirow{4}{*}{$\begin{array}{c}\theta_{\mathrm{v}}=0.73 \times \theta_{\mathrm{vi}}+13.75 \\
\theta_{\mathrm{v}}=17.01 \times \mathrm{e}^{0.025 \times \theta_{\mathrm{vi}}} \\
\theta_{\mathrm{v}}=17.64 \times \ln \left(\theta_{\mathrm{vi}}\right)-24.3 \\
\theta_{\mathrm{v}}=-0.051 \times\left(\theta_{\mathrm{vi}}\right)^{2}+3.19 \times \\
\theta_{\mathrm{vi}}-14.39\end{array}$} & -0.0011 & 2.21 & 0.66 \\
\hline & Exponential & & -0.10 & 2.42 & 0.63 \\
\hline & Logarithmic & & 0.00054 & 1.96 & 0.74 \\
\hline & Quadratic & & -0.012 & 1.66 & 0.81 \\
\hline \multicolumn{3}{|c|}{ Proposed evaluation criteria } & \pm 1.0 & $<3.5$ & $>0.65$ \\
\hline
\end{tabular}

$\theta_{\mathrm{vi}}$ is factory calibrated $\theta_{\mathrm{v}}$ as an input in percent $(\%)$ and not directly as a fraction $\left(\mathrm{m}^{3} \mathrm{~m}^{-3}\right)$. MD indicates mean difference. RMSE indicates root mean square error.

As for the relationship between correction equations and installation orientations, the DEC-GS1 sensors using the horizontal and vertical insertions had the closest equation coefficients (" $\mathrm{a}$ " and " $\mathrm{b}$ ") when using the linear and exponential equation types (Table 5). However, small $R^{2}$ values were evident with the horizontal insertion orientation. The relatively short electrodes of these sensors may affect the "integrating potential" as compared to sensors with longer waveguides. The apparent differences of the 
equation coefficients are shown for all equation types when comparing CS-655 sensors using horizontal insertion against vertical insertion. Regarding the WM-200SS sensors, the three installation orientations exhibited close equation coefficients for all equation types. Overall, the ACC-315 L, DEC-GS1, and CS-655 sensors were sensitive to installation orientation, and different field correction equations may need to be used with the respective installation orientation. As shown in Table 5, the WM-200SS sensor was not susceptible to installation orientation, which provides flexibility in sensor installation. However, field-specific correction is required for agricultural applications. We acknowledge there are some limitations of this study. It is generally suggested that installation orientation should not affect sensor readings, but data in this study using these installation processes suggested otherwise.

These are preliminary data from the first year of the study, and the disturbed soil (due to installation) may need time to consolidate. It may also require a "water packing effect (generally achieved through multiple irrigations or heavy rains)" to adequately settle the soil in the trench even though the sensors were installed in February 2018. While this settling period is recognized and necessary, installation according to the manufacturer's recommendations would also require a bore hole or equivalent entry port to install the sensors in the horizontal orientation, particularly at deeper profile depths. In addition, the spatial and depth matching of the sensor and NMM measurements have a computational impact on the comparison values on the soil water in a management layer. Finally, direct calibration of the sensor-measured apparent permittivity may be necessary when more data are available from subsequent study years. This applied research addresses how actual producers could use the soil water sensors for irrigation scheduling, which may or may not follow manufacturer recommendations regarding the installation, interpretation, and use of sensor readings. The results in this study provide information of the performance of various soil water sensors under different irrigation treatments with multiple installation orientations and offer soil-specific correction equations for the heavier soils of the Texas High Plains.

\section{Conclusions}

This study evaluated the performance of ACC-315L, DEC-GS1, CS-655, and WM-200SS soil sensors in multiple field-based water regimes between PWP and FC using various installation orientations with the factory calibration and field correction in a Pullman clay loam soil using NMM readings as a control reference. Overall, good agreement between sensor measurements and NMM readings was observed at the depths of 45.7 and $76.2 \mathrm{~cm}$ under the three irrigation treatments. Sensor installation orientation had a large impact on the performance of most sensors. In general, the horizontal and vertical insertions with less soil disturbance yielded sensor readings that were more representative of field water contents compared with the laid horizontal orientation. The factory-calibrated ACC-315L performed satisfactorily using the horizontal insertion installation at depths of 45.7 and $76.2 \mathrm{~cm}$ with values of RMSE $<3.5 \%, \mathrm{SD}<2.4 \%$, and $\mathrm{MD}$ within $\pm 2 \%$. Also, it performed optimally in terms of soil profile water storage using a horizontal insertion orientation across all irrigation treatments $(\mathrm{RMSE}=29.25 \mathrm{~mm}$ or $3.25 \%$ and $\mathrm{MD}=3.27 \mathrm{~mm}$ or $0.36 \%$ ). Satisfactory performance of the factory-calibrated ACC-315L sensors with a horizontal insertion orientation can be used and benefit local/regional producers, who may not be willing to use a field correction. This research also suggests that field correction is required for the DEC-GS1, CS-655, and WM-200SS sensors to be used in irrigation scheduling. One linear and three non-linear field correction equations were reported for possible use by crop/irrigation consultants, extension professionals, and relatively experienced soil water sensor users and producers to correct the soil water sensors for use in regions with a similar soil. Finally, according to the sensor comparison of depths and comparison of soil profile water storage, we suggest the sensor performance statistical goals of MD within $\pm 2 \%$, RMSE $<3.5 \%$, and $R^{2}>0.65$ for data fitting can be used in specific depth comparisons. As for the comparison of soil profile water storage, the evaluation criteria for sensor performance of MD within $\pm 1 \%$, RMSE $<3.5 \%$, and $R^{2}>0.65$ for data fitting is recommended in most agricultural and research applications. This sensor performance assessment and correction study also serves to potentially provide statistically-based soil water sensor 
recommendations for the USDA-NRCS agency guidelines as well as that of other water management and water policy agencies.

Supplementary Materials: The following is available online at http://www.mdpi.com/1424-8220/19/13/2872/s1, Table S1: Soil information for Pullman clay loam soil at Bushland, TX, USA, Figure S1: Illustration of the multiple sensor installation orientations including laid horizontal, vertical insertion, and horizontal insertion at the study site. Note the extensive amount of wiring leads in the trench with the sensor array., Figure S2: Graphical and statistical comparisons of sensor and neutron moisture meter-derived soil profile water storage values in the upper $0.9 \mathrm{~m}$ soil profile under the $100 \%$ crop evapotranspiration treatment. MD indicates mean difference; RMSE indicates root mean square error; ${ }^{*}$ indicates a significant difference at $p<0.05$; ${ }^{* *}$ indicates a significant difference at $p<0.01$., Descriptions of Neutron Moisture Meter and Soil Water Sensors.

Author Contributions: Conceptualization, T.H.M., D.O.P., G.W.M., and Y.C.; methodology, Y.C., G.W.M., T.H.M., K.R.H., D.O.P., J.E.M., and D.K.B.; formal analysis, Y.C., G.W.M., and T.H.M.; investigation, Y.C., G.W.M., and T.H.M.; resources, G.W.M., T.H.M., K.R.H., D.O.P., and D.K.B.; data curation, Y.C., G.W.M., T.H.M., K.R.H., and J.E.M.; writing-original draft preparation, Y.C., G.W.M., and T.H.M.; writing - review and editing, K.R.H., D.O.P., J.E.M., and D.K.B.; visualization, Y.C., G.W.M., and T.H.M.; supervision, G.W.M., T.H.M., D.O.P., and D.K.B.; project administration, G.W.M., T.H.M., D.O.P., and D.K.B.; funding acquisition, G.W.M., T.H.M., D.O.P., and D.K.B.

Funding: This research was funded by the Natural Resources Conservation Service, U.S. Department of Agriculture, under grant No. 69-3A75-16-013. It was also supported by university-based state Hatch projects included in the REEport system.

Acknowledgments: This research was supported in part by the Ogallala Aquifer Program, a consortium between USDA-Agricultural Research Service, Kansas State University, Texas A\&M AgriLife Research, Texas A\&M AgriLife Extension Service, Texas Tech University, and West Texas A\&M University.

Disclaimer: The use of trade, corporation, and firm names in this paper is for informational purposes only and are included for the convenience of the readers. Texas A\&M University System and the U.S. Department of Agriculture-Agricultural Research Service neither endorse nor approve the suitability of any brand or use of commercial products from these companies.

Conflicts of Interest: The authors declare no conflict of interest.

\section{References}

1. Colaizzi, P.D.; Gowda, P.H.; Marek, T.H.; Porter, D.O. Irrigation in the Texas High Plains: A brief history and potential reductions in demand. Irrig. Drain. 2008, 58, 257-274. [CrossRef]

2. Marek, T.H.; Amosson, S.H.; Bretz, F.E.; Guerrero, B.; Kotara, R. 2011 Panhandle regional water plan task 2 report: Agricultural water demand projections. In Technical Report for Texas Water Development Board and Region A Panhandle Regional Planning Group through Freese and Nichols, Inc. AREC \#09-21; Texas AgriLife Research and Extension Center: Amarillo, TX, USA, 2009; p. 83.

3. Claeys, H.; Inzé, D. The agony of choice: How plants balance growth and survival under water-limiting conditions. Plant Physiol. 2013, 162, 1768-1779. [CrossRef] [PubMed]

4. Charlesworth, P.; Stirzaker, R.J. Irrigation Scheduling by Soil Water Status. In Encyclopedia of Water Science; Stewart, B.A., Howell, T.A., Eds.; Marcel Dekker, Inc.: New York, NY, USA, 2003; pp. 528-531.

5. National Resource Conservation Service (NRCS), Agricultural Water Enhancement Program Project Proposals Approved for Fiscal Year 2009; U.S. Department of Agriculture: Washington, DC, USA. Available online: http://www.nrcs.usda.gov/programs/awep/2009projects.html (accessed on 25 March 2019).

6. Caldwell, T.G.; Bongiovanni, T.; Cosh, M.H.; Halley, C.; Young, M.H. Field and laboratory evaluation of the CS655 soil water content sensor. Vadose Zone J. 2018, 17, 170214. [CrossRef]

7. Mohanty, B.P.; Cosh, M.H.; Lakshmi, V.; Montzka, C. Soil moisture remote sensing: State-of-the-science. Vadose Zone J. 2017, 16. [CrossRef]

8. Hignett, C.; Evett, S. Direct and surrogate measures of soil water content. In Field Estimation of Soil Water Content: A Practical Guide to Methods, Instrumentation, and Sensor Technology; Evett, S.R., Heng, L.K., Moutonnet, P., Nguyen, M.L., Eds.; IAEA-TCS-30; International Atomic Energy Agency: Vienna, Austria, 2008; ISSN 1018-5518.

9. Seyfried, M.S.; Grant, L.E.; Du, E.; Humes, K. Dielectric loss and calibration of the Hydra Probe soil water sensor. Vadose Zone J. 2005, 4, 1070-1079. [CrossRef]

10. Evett, S.R.; Tolk, J.A.; Howell, T.A. Soil profile water content determination: Sensor accuracy, axial response, calibration, temperature dependence, and precision. Vadose Zone J. 2006, 5, 894-907. [CrossRef] 
11. Paige, G.B.; Keefer, T.O. Comparison of field performance of multiple soil moisture sensors in a semi-arid rangeland. J. Am. Water Resour. Assoc. 2008, 44, 121-135. [CrossRef]

12. Kammerer, G.; Nolze, R.; Rodney, M.; Loiskandl, W. Performance of Hydra Probe and MPS-1 soil water sensors in tropical tested in lab and field. J. Water Resour. Prot. 2014, 6, 1207-1219. [CrossRef]

13. Ojo, E.R.; Bullock, P.R.; Fitzmaurice, J. Field performance of five soil moisture instruments in heavy clay soils. Soil Sci. Soc. Am. J. 2015, 79, 20-29.

14. Plauborg, F.; Iversen, B.V.; Lærke, P.E. In situ comparison of three dielectric soil moisture sensors in drip irrigated sandy soils. Vadose Zone J. 2005, 4, 1037-1047. [CrossRef]

15. Chow, L.; Xing, Z.; Rees, H.W.; Meng, F.; Monteith, J.; Stevens, L. Field performance of nine soil water content sensors on a sandy loam soil in New Brunswick, Maritime Region, Canada. Sensors 2009, 9, 9398-9413. [CrossRef] [PubMed]

16. Klocke, N.L.; Currie, R.S.; Tomsicek, D.J.; Koehn, J. Corn yield response to deficit irrigation. Trans. ASABE 2011, 54, 931-940. [CrossRef]

17. Hao, B.; Xue, Q.; Marek, T.H.; Jessup, K.E.; Hou, X.; Xu, W.; Bynum, E.D.; Bean, B.W. Soil water extraction, water use, and grain yield by drought-tolerant maize on the Texas High Plains. Agric. Water Manag. 2015, 155, 11-21. [CrossRef]

18. Howell, T.A.; Copeland, K.S.; Schneider, A.D.; Dusek, D.A. Sprinkler irrigation management for corn-southern great plains. Trans. ASABE 1989, 32, 147-155. [CrossRef]

19. Schlegel, A.J.; Assefa, Y.; O’Brien, D.; Lamm, F.R.; Haag, L.A.; Stone, L.R. Comparison of corn, grain sorghum, soybean, and sunflower under limited irrigation. Agron. J. 2016, 108, 670-679. [CrossRef]

20. Trout, T.J.; Bausch, W.C. USDA-ARS Colorado maize water productivity data set. Irrig. Sci. 2017, 35, $241-249$. [CrossRef]

21. Varble, J.L.; Chávez, J.L. Performance evaluation and calibration of soil water content and potential sensors for agricultural soils in eastern Colorado. Agric. Water Manag. 2011, 101, 93-106. [CrossRef]

22. Unger, P.W.; Pringle, F.B. Pullman Soils: Distribution Importance, Variability, and Management; Bulletin B-1372; Texas Agricultural Experiment Station: College Station, TX, USA, 1981.

23. Heng, L.K.; Hsiao, T.; Evett, S.; Howell, T.; Steduto, P. Validating the FAO AquaCrop model for irrigated and water deficient field maize. Agron. J. 2009, 101, 488-498. [CrossRef]

24. Tolk, J.A.; Evett, S.R. Lower limits of crop water use in three soil textural classes. Soil Sci. Soc. Am. J. 2012, 76, 607-616. [CrossRef]

25. American Society of Civil Engineers-Environmental \& Water Resources Institute (ASCE-EWRI). The ASCE Standardized Reference Evapotranspiration Equation; Technical Committee report to the Environmental and Water Resources Institute of the American Society of Civil Engineers from the Task Committee on Standardization of Reference Evapotranspiration; ASCE-EWRI, 1801 Alexander Bell Drive: Reston, VA, USA, 2005; p. 173.

26. Bell, J.P.; Dean, T.J.; Hodnett, G.C. Soil moisture measurement by an improved capacitance technique, Part II. Field techniques, evaluation and calibration. J. Hydrol. 1987, 93, 79-90. [CrossRef]

27. Schwartz, R.C.; Evett, S.R.; Lascano, R.J. Comments on J. Singh et al., performance assessment of factory and field calibrations for electromagnetic sensors in a loam soil. Agric. Water Manag. 2018, 196, 87-98.

28. Evett, S.R.; Howell, T.A.; Steiner, J.L.; Cresap, J.L. Evapotranspiration by soil water balance using TDR and neutron scattering. In Management of Irrigation and Drainage Systems, Integrated Perspectives; Allen, R.G., Neale, C.M.U., Eds.; American Society of Civil Engineers: New York, NY, USA, 1993; pp. 914-921.

29. Addiscott, T.M.; Whitmore, A.P. Computer simulation of changes in soil mineral nitrogen and crop nitrogen during autumn, winter and spring. J. Agric. Sci. 1987, 109, 141-157. [CrossRef]

30. Loague, K.; Green, R.E. Statistical and graphical methods for evaluating solute transport models: Overview and application. J. Contam. Hydrol. 1991, 7, 51-73. [CrossRef]

31. Bland, J.M.; Altman, D.G. Statistics notes: Measurement error. BMJ 1996, 312, 1654. [CrossRef] [PubMed]

32. De willigen, P.; Neeteson, J.J. Comparison of six simulation models for the nitrogen cycle in the soil. Fert. Res. 1985, 8, 157-171. [CrossRef]

33. Wonnacott, T.H.; Wonnacott, R.J. Introductory Statistics; John Wiley \& Sons: New York, NY, USA, 1969; pp. 234-254.

34. Schwartz, R.C.; Baumhardt, R.L.; Evett, S.R. Tillage effects on soil water redistribution and bare soil evaporation throughout a season. Soil Tillage Res. 2010, 110, 221-229. [CrossRef] 
35. Datta, S.; Taghvaeian, S.; Ochsner, T.E.; Moriasi, D.; Gowda, P.H.; Steiner, J. Performance assessment of five different soil moisture sensors under irrigated field conditions in Oklahoma. Sensors 2018, 18, 3786. [CrossRef] [PubMed]

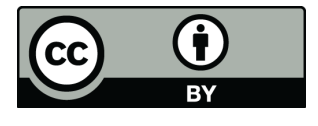

(C) 2019 by the authors. Licensee MDPI, Basel, Switzerland. This article is an open access article distributed under the terms and conditions of the Creative Commons Attribution (CC BY) license (http://creativecommons.org/licenses/by/4.0/). 